\title{
Apendicitis crónica en un apéndice atrófico: reporte de un caso
}

\author{
Yeray Trujillo L ${ }^{1}$, Carlos Contreras $C^{2, a, b}$, José Cabanillas $L^{3}$
}

\section{RESUMEN}

La apendicitis crónica es una entidad poco frecuente que se caracteriza por dolor en fosa iliaca derecha durante 2 años de evolución. Algunos cirujanos y patólogos niegan la existencia de ésta enfermedad, contrariamente, existen estudios de diversos autores que reportan hallazgos de apendicitis crónica.

Paciente varón de 71 años de edad, con dolor en fosa iliaca derecha de 2 semanas de evolución que remitía parcialmente a lo largo de su presentación, no asociado a náuseas, vómitos ni anorexia.

El examen físico reveló rebote en fosa iliaca derecha y hemograma sin leucocitosis y las formas segmentadas dentro de parámetros normales. Antecedente de 2 años de cuadros similares con resolución espontánea. Se decide realizar laparotomía exploratoria, el hallazgo operatorio fue apéndice cecal de $1 \mathrm{~cm}$ de longitud con adherencia de su extremo distal a la pared abdominal, ejerciendo tracción. En el postoperatorio el paciente cursó con leve distensión abdominal y remisión completa del dolor. La anatomía patológica de la pieza operatoria fue informada como apéndice cecal de $1 \mathrm{~cm}$ de longitud con inflamación crónica y fibrosis.

Palabras clave: apendicitis, apéndice, apendicectomía.

\section{Chronic appendicitis in an atrophic appendix: case report}

\begin{abstract}
Chronic appendicitis is a rare entity characterized by pain in the right iliac Fossa during 2 years of evolution. Some surgeons and pathologists deny the existence of this disease; in contrast, there are studies of different authors that reported findings of chronic appendicitis.

Male patient, 71 years- old, with pain in the right iliac Fossa of 2 weeks of evolution which remitted partially throughout his presentation, not associated with nausea, vomiting or anorexia. Physical examination revealed bounce in right iliac Fossa and blood count without Leukocytosis and forms segmented within normal parameters. History of 2 years of similar symptoms with resolution spontaneously. It was decided to carry out exploratory laparotomy; the operative finding was a cecal appendix of $1 \mathrm{~cm}$ of length with adherence of its distal part to the abdominal wall, exerting traction. Postoperatively the patient presented mild bloating and complete remission of pain. The pathological anatomy of the operative part was informed as cecal appendix $1 \mathrm{~cm}$ of length with chronic inflammation and fibrosis.
\end{abstract}

Key words: appendicitis, appendix, appendectomy.

1. Servicio de Cirugía General del Hospital Nacional Dos de Mayo. Lima - Perú. Docente de Anatomía Humana UNMSM. Lima, Perú.

2. Departamento de Emergencia y Cuidados Críticos del Hospital Nacional Dos de Mayo, Lima - Perú.

3. Servicio de Anatomía Patológica del Hospital Nacional Dos de Mayo, Lima - Perú.

a. Docente de la USMP, Lima - Perú.

b. Instituto de Investigaciones Clínicas, Universidad Nacional Mayor de San Marcos, Lima, Perú. 


\section{INTRODUCCIÓN}

La apendicitis crónica es una entidad poco frecuente que se caracteriza por dolor recurrente en la fosa ilíaca derecha durante un tiempo promedio de 2 años de evolución ${ }^{(1)}$, se asocia frecuentemente a sintomatología atípica, puede o no presentar náuseas, vómitos, anorexia, elevación de temperatura, migración del dolor y rebote y la mayoría no presentan leucocitosis ni incremento de segmentados $^{(2,3)}$. Esta entidad se sospecha frente a la presencia de un cuadro atípico, dolor recurrente y remisión del cuadro tras la apendicectomía. Investigaciones realizadas en Colombia y en México, destacan la presencia de criterios diagnósticos del mismo confirmándose con cambios inflamatorios crónicos en la anatomía patológica ${ }^{(4-6)}$.

Sgourakis G. et al, destacaron importantes aspectos fisiopatológicos en sus investigaciones realizadas sobre apendicitis crónica, determinaron que esta entidad no solo está asociada a la presencia de coprolitos, sino, que también puede estar relacionada a la coprostasia ${ }^{(7)}$.

A pesar de la infrecuente presentación de la apendicitis crónica y la probable inexistencia de esta entidad afirmada por algunos cirujanos y patólogos, existen numerosas investigaciones que corroboran la existencia de la apendicitis crónica demostrada por estudios anatomopatológicos ${ }^{(2,5,8,9,10)}$.

\section{CASO CLÍNICO}

Paciente varón de 71 años, natural y procedente de Lima, acude a emergencia refiriendo dolor en fosa iliaca derecha de 4 días de evolución y que se inició como un dolor abdominal difuso, el dolor incrementó en intensidad progresivamente agregándose luego, anorexia refirió ausencia de náuseas, vómitos y se mantuvo afebril durante la hospitalización.

A su vez manifestó que desde hace 2 años presentó episodios de dolor similares y hace 2 semanas presentó cuadro similar que cedió espontáneamente.

Al examen físico presentaba el abdomen blando, depresible, Mc Burney (+), Blumberg (+). El hemograma no presentaba leucocitosis $(5700$ leucocitos), no hubo incremento de segmentados (44\%), no se evidenció incremento de abastonados $(0 \%)$. Ingresó a sala de operaciones con el diagnóstico de apendicitis aguda. El diagnóstico posoperatorio fue: Atrofia de apéndice y brida apendicular.
En su primer día post operatorio cursa con distensión abdominal que cede al día siguiente, se le observa hasta el cuarto día post operatorio que es dado de alta. El dolor en fosa iliaca derecha cede en su totalidad.

La anatomía patológica del paciente reveló Apéndice cecal de $1 \mathrm{~cm}$ con luz fibrosada (Figura 1). Escasa presencia de tejido linfoideo, presencia de tejido adiposo e inflamación crónica (Figura 2).

Presencia de tejido fibroconjuntivo que se encuentra reemplazando al tejido muscular, característico de las patologías crónicas (Figura 3). Ausencia casi total de la luz apendicular por la naturaleza atrófica de la misma así como, presencia de tejido fibroso (Figura 4), no se encontraron cambios morfológicos de atipia celular. Todos estos hallazgos encontrados en el estudio anatomopatológico son compatibles con apendicitis crónica.

Los hallazgos intraoperatorios fueron (Figura 5):

- Apéndice cecal de aproximadamente $1 \mathrm{~cm}$ de longitud sin signos inflamatorios cuyo extremo distal es traccionado hacia colon ascendente por brida.

- Resto de órganos intra abdominales sin alteraciones.

Figura 1. Vista panorámica, corte transversal de apéndice cecal

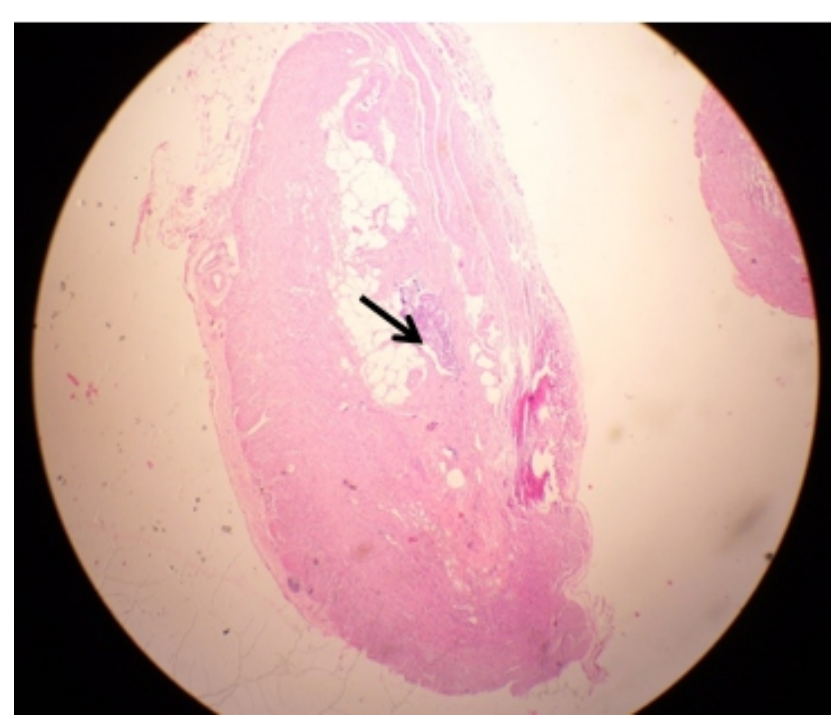

Se observa ausencia casi total de luz apendicular (flecha) alrededor del cual se observa tejido muscular remanente con tendencia a la fibrosis, la serosa no muestra mayor alteración. 
Figura 2. Inflamación crónica apendicular

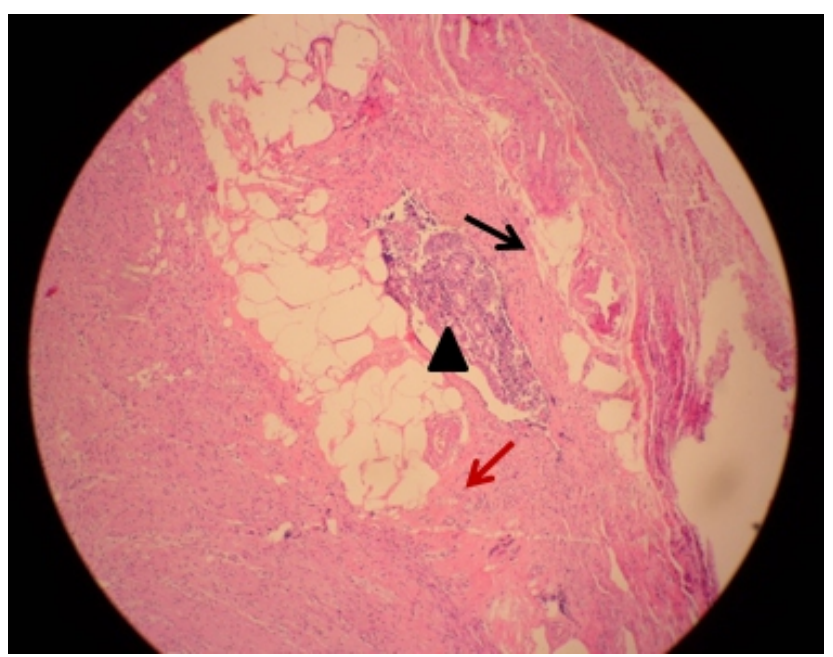

La flecha negra señala rezagos de tejido linfoide asociado a la mucosa apendicular, alrededor se observa cambios fibrosos asociados a inflamación crónica no activa. En el lado contralateral con flecha roja ausencia total de tejido linfoide (atrofia). El triángulo señala la escasa luz apendicular.

Figura 3. Inflamación crónica y atrofia apendicular

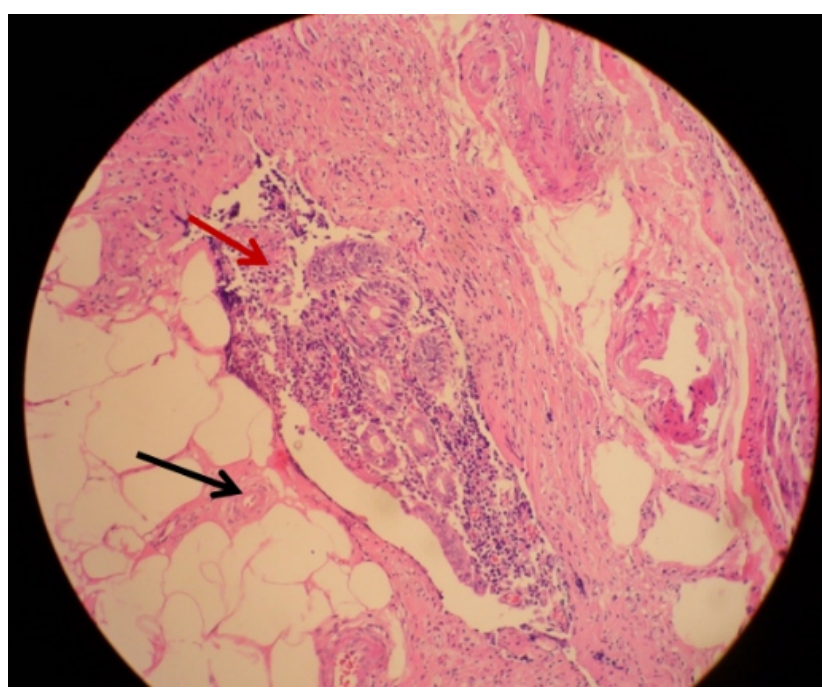

A mayor aumento resalta el espacio correspondiente a la capa muscular que ha sido reemplazado parcialmente por tejido fibroadiposo (flecha negra) y alrededor del mismo, tejido fibroconjuntivo que reemplaza parcialmente al tejido muscular con remanente inflamatorio crónico en un extremo (flecha roja).
Figura 4. Vista panorámica corte proximal de apéndice cecal

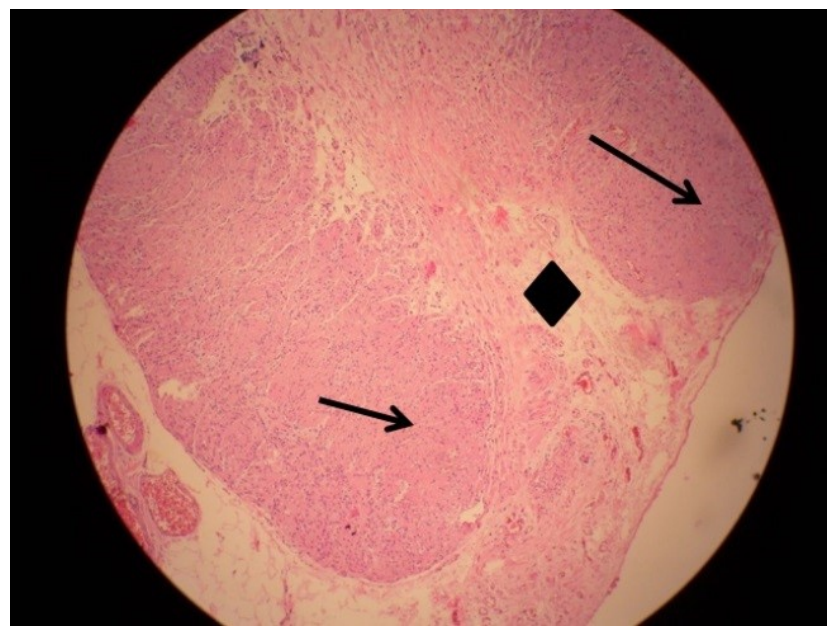

En este corte más proximal del apéndice se observa la ausencia total de la luz apendicular (flecha negra grande), reemplazado parcialmente por tejido fibroadiposo (cuadrado negro). La flecha negra pequeña, señala el tejido muscular adyacente.

\section{DISCUSIÓN}

El caso presentado describe el cuadro clínico de una apendicitis crónica que como se mencionó previamente su existencia para algunos cirujanos está aún en debate. Sin embargo, se encuentra en la literatura casos cuyo cuadro clínico coincide y que se corrobora con cambios inflamatorios crónicos en la anatomía patológica ${ }^{(11-13)}$. Adicionalmente en el acto operatorio se encontró una brida apendicular el cual ejerce tracción del mismo hacia colon ascendente, éste hallazgo coincide con las descripciones de otros autores que según se indica se explicaría por los cuadros de dolor a repetición que presentan los pacientes ${ }^{(14)}$.

Por otro lado, en la mayoría de la literatura no se describe la existencia de apéndices atróficos, pero si se describen casos de agenesia apendicular, duplicación apendicular, etc. que incluso se las ha incluido dentro de la clasificación de Collins ${ }^{(15)}$. Si bien es cierto la apendicitis crónica es infrecuente, la presentación de ésta en un apéndice atrófico es extremadamente infrecuente.

Entre otras variantes anatómicas del apéndice cecal se encuentran reportes que describen la duplicación apendicular que generalmente es asintomático, y se presenta en adultos. La duplicación apendicular suele ser un hallazgo incidental en el curso de una 
laparotomía o en estudios de imágenes. Se reportan de 60 a 80 casos desde que fue descrito inicialmente en $1903^{(16-20)}$. El año 2011 se publicó en la Revista Cubana de cirugía el reporte de un caso de una mujer de 31 años con cuadro clínico típico de apendicitis aguda. Se encontraron dos apéndices cecales uno de implantación habitual y otro en uno de las tenias a 2 $\mathrm{cm}$ del primero, además que ambos tenían luz y mesoapéndice propio ${ }^{(21)}$.

Otra variante más en la presentación del apéndice cecal es la agenesia apendicular que fue estudiada ampliamente el año 1974 por Collins ${ }^{(22)}$ donde estudió 50 mil laparotomías identificando 8 malformaciones apendiculares, 4 casos de agenesia y 4 duplicaciones parciales o totales. La clasificación propuesta por Collins para agrupar las malformaciones apendiculares fue la siguiente: Tipo I: Ausencia completa de apéndice y ciego. Tipo II: Ciego rudimentario y ausencia de apéndice. Tipo III: Ciego normal sin apéndice. Tipo IV: Ciego normal y apéndice rudimentario y Tipo V: Ciego gigante sin apéndice.

Se describen dos reportes de caso, uno de un hombre de 48 años de edad con diagnóstico presuntivo de apendicitis aguda y que luego de realizada la intervención quirúrgica se le diagnostica con adenitis mesentérica y con una malformación apendicular tipo IV de Collins ${ }^{(23)}$. Otro caso reportado es también de un paciente varón de 39 años de edad con un tiempo de enfermedad de 72 horas compatible con apendicitis aguda, al ingresar a sala de operaciones encuentran un ciego subhepático y una adenitis mesentérica sin hallarse el apéndice cecal, aunque en el reporte no se describe el tipo de malformación apendicular ésta correspondería a un tipo III de Collins que se describe como el más frecuente en la revisión de la literatura ${ }^{(24)}$.

El término atrofia se refiere a la disminución del tamaño de la célula por la pérdida de sustancia celular y cuando participan un número suficiente de células todo el órgano disminuye de tamaño y se hace atrófico, a su vez se sabe que cada órgano tiene una longitud y tamaño conocido dentro de márgenes normales y que cuando se encuentra éste órgano por debajo de los límites normales se considera atrofia ${ }^{25)}$.

Además, sabemos que la longitud del apéndice cecal es de 6 a $10 \mathrm{~cm}$. por debajo del límite inferior se debe considerar atrofia apendicular. El cual coincidiría con la malformación Tipo IV de Collins con la diferencia que éste autor no lo llama apéndice atrófico si no apéndice rudimentario).
El hallazgo de estas dos características anatómicas encontradas en el presente caso clínico (apendicitis crónica y apéndice atrófico), revela la trascendencia caso clínico, así mismo, debido a la escaza bibliografía y la polémica que suscita la presencia de apendicitis crónica, se ha propuesto la publicación de este caso.

Figura 5. Atrofia apendicular

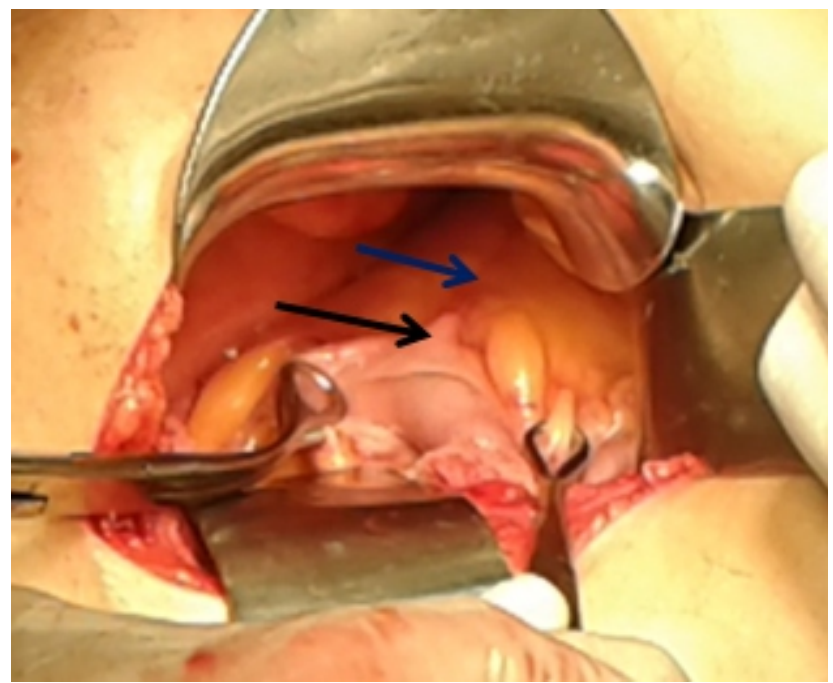

Se muestra una fotografía del acto intraoperatorio donde se observa el apéndice cecal de tamaño pequeño (flecha negra) rodeado por abundante tejido graso.

En la parte superior (flecha azul) se observa la presencia de una brida que tracciona el apéndice cecal a la pared abdominal.

\section{REFERENCIAS BIBLIOGRÁFICAS}

1. Shah S, Gaffney R. Chronic Appendicitis: An Often Forgotten Cause of Recurrent Abdominal Pain. The American Journal of Medicine. 2013; 126 (1): 7-8.

2. Kemal P, Kemal K. A Case of Chronic Appendicitis. Kafkas J Med Sci 2012; 2(2):78-80.

3. Lai DH, Chuang CH, Yu JC, Hsieh CB, Wu HS, Lin CH. Chronic or recurrent appendicitis? Rev esp Enferm Dig (Madrid). 2007; 99 (10): 613-621.

4. Zundel N, Albis R. Apendicitis crónica $y / o$ apendicitis recurrente? Rev. Col. Cir. 1992; 7 (1): 38-42.

5. Montiel AJ, Gómez E, Reyes P, Romero C, Mendoza AV, García UN. Apendicitis crónica. Caso clínico. Rev Med Inst Mex Seguro Soc 2008; 46 (4): 431-434.

6. Ali Sheikh I, ShoaibHanif M. Recurrent Acute / Chronic Appendicitis an Independent Clinical Entity. A Journal of Army medical Corps [internet] 2005 (Citado en febrero 2014); 4. Disponible en: http://www.pafmj.org/showdetails.php?id $=71 \& \mathrm{t}=0$

7. Sgourakis G, Sotiropoulos GS, Molmenti EP, Eibl C, Bonticous $\mathrm{S}$, Moege $\mathrm{J}$ et al. Are acute exacerbations of chronic 
inflammatory appendicitis triggered by coprostasis and/or coproliths? World J Gastroenterol. 2008; 14(20): 3179-3182.

8. Safaei M, Moeinei L, Rasti M. Recurrent Abdominal Pain and Chronic Appendicitis. J Res Med Sci. 2013. 2004; 1: 11-14.

9. Mussack T, Schmidbauer S, Nerlich A, Schdmidt W, Hallfeldt KK.Die chronischeAppendizitisalseigenständigeklinische Entität.DerChirug. 2002; 73 (7): 210-215

10. Al-Araji KS, Recurrent "Chronic"Appendicitis ; Is it a myth ? Is Appendicectomy Indicated?Medical Journal of Babylon. 2006; 3 $(1,2): 51-55$

11. López ND, Domínguez H, Castillo L, Paseiro R, Umpíerre I, Alfonso 0 . Apendicitis Crónica. A propósito de un paciente. Scielo Rev. Med. Electrón. [internet]. 2009 [citado marzo 2009] ; 31 (2). Disponible en: http://scielo.sld.cu/scielo. php?script=sci_pdf\&pid=S1684-18242009000200014\&lng =es\&nrm=iso\&tlng=es

12. Sazhin AV, Mosin SV, Kodzhoglian AA, Mirozoian AT, Laipanov BK. Epidemiological aspects of chronic appendicitis. Khirurgiia (Mosk). 2011; (4): 4-8.

13. Sazhin AV, Mosin SV. Clinical and morphological aspects of chronic appendicitis. Khirurgiia (Mosk).2007; (12) 59-64.

14. Ramírez Chacón JL. Apendicitis crónica como causa del dolor Crónico en fosa iliaca derecha. Revista Médica de la Universidad de Costa Rica. 2012; 6 (2): 30-36.

15. Zetina CA, Álvarez JE, Quillo J. Agenesia del apéndice cecal. Informe de un caso. Cir Ciruj 2009;77 (5):407-410.

16. Eroglu E, Erdogan E, Gundogdu G, Dervisoglu S, Yeker D. Duplication of appendix vermiformis: a case in a child. Tech Coloproctol. 2002; 6:55-7.

17. Pratt HN. Double appendix associated with other congenital anomalies. Am J Dis Children. 1933; 45:1263.
18. Aitkens AB. Case of doubling of the great intestine. Glasgow Med J. 1912;76:431.

19. Cave AJE. Appendix vermiformis duplex. J Anat. 1936;70:283.

20. Berthold F. The occurrence of a double appendix. Centralbi F. Chirurgie. 1932;59:2935

21. Taquechel Barreto F, Cruz Alonso J. Duplicación apendicular. Revista Cubana de Cirugía. 2011;50(3):348-352.

22. Collins DC. A study of 50,000 specimens of the human vermiform appendix. Am J Surg. 1974;127:704-707.

23. Garteiz Martínez D, Weber Sánchez A. Agenesia apendicular. Acta Médica Grupo Ángeles. 2010; 8:167-171.

24. Kumar V. Patología Humana. 9 ed. Madrid: Elsevier; 2015.

25. Skandalakis J. Cirugía.14 ed. Madrid: Marbán Libros; 2013.

\section{Fuentes de financiamiento}

Este artículo ha sido financiado por los autores.

\section{Conflictos de interés}

Los autores declaran no presentar ningún conflicto de interés.

\section{Correspondencia:}

Carlos Contreras Camarena

Dirección: Jr. Trinidad Moran 275 Urb.El Retablo I Etapa. Lima 07 Teléfono: 945933754

Correo electrónico: ccontrerashndm@hotmail.com

Recibido: 26 de abril de 2016 Aprobado: 24 de junio de 2016 